\title{
Citrullinemia with an atypical presentation: persistent hiccups. Case report
}

\author{
Halil Degirmencioglu M.D. ${ }^{a}$, Mehmet Yekta Oncel M.D. ${ }^{a}$, Sadik Yurttutan M.D. ${ }^{b}$, Sadrettin Ekmen M.D. ${ }^{a}$, \\ Serife Suna Oguz M.D. ${ }^{a}$, Nurdan Uras M.D. ${ }^{a}$ and Ugur Dilmen M.D. ${ }^{a, c}$
}

\begin{abstract}
We report an infant who developed encephalopathy within the first 3 days of life. He had persistent hiccups that progressed to deep coma 72 hours after admission. The sepsis parameters and cerebrospinal fluid examination (CSF) were normal. The metabolic evaluation confirmed hyperammonemia, and hypercitrullinemia. The ratio of CSF/plasma glycine concentration was normal. This did not agree with our initial diagnosis of nonketotic hyperglycinemia where hiccups is present more often.

Neonatal onset of argininosuccinic acid synthetase deficiency (ASD; citrullinemia) should be brought in mind in the differential diagnosis of encephalopathy in association with hiccups in the neonatal period suggesting inborn errors of metabolism.
\end{abstract}

Key words: citrullinemia, hiccups, neonate.

http:/ / dx.doi.org/10.5546/aap.2014.eng.e206

\section{INTRODUCTION}

Citrullinemia is a rare autosomal recessive inborn error of the urea cycle metabolism, caused by a deficient argininosuccinic acid synthetase, with an estimated incidence of $1 / 57,000$ live births. ${ }^{1}$ It occurs in three clinical forms (neonatal, infantile, and adult) and three types according to the enzymatic anomaly. ${ }^{2}$ Severe hyperammonemia and hypercitrullinemia may lead to neurological deterioration in citrullinemia. ${ }^{3,4}$

Hiccups are usually benign but is considered "persistent" if they last more than 48 hours up to one month; when they continue more than a month are considered "intractable" and can lead to complications. ${ }^{5}$

a. Division of Neonatology, Zekai Tahir Burak Maternity Training Hospital, Ankara, Turkey.

b. Department of Pediatrics, Sutcu Imam University School of Medicine, Kahramanmaras, Turkey.

c. Department of Pediatrics, Yildirim Beyazit University School of Medicine, Ankara, Turkey.

E-mail Address:

Halil Degirmencioglu M.D.: hdegirmencioglu@gmail.com

Conflict of interest: none.

Received: 12-30-2013

Accepted: 4-9-2014
To the best of our knowledge encephalopathy in association with persistent hiccups in the neonatal period has not been reported with citrullinemia. We report a case of citrullinemia in a newborn with this clinical manifestations.

\section{CASE REPORT}

A male infant was brought with complaints of poor feeding, vomiting, lethargy and remarkable hypoactivity by parents at 3 days of life. The infant was normal at birth and was feeding well during the first two days of life. He had difficulty of feeding and became lethargic with persistent hiccups on the third day of life. He was born by caesarean section at our hospital at 39 weeks 4 days' gestation without pregnancy complications to a 40-year-old fourth gravida mother. His birth weight was $3030 \mathrm{~g}$; Apgar score was 7-9 at one and five minutes, respectively. There was no history of consanguinity and a fetal missing was recorded. The other siblings were healthy. Physical examination revealed no dysmorphic features or anomalies. Firstly, he had increased muscle tone and hyperreflexia in both right upper and lower extremities, with persistent hiccups. Subsequently he had generalized hypotonia, respiratory distress and rapidly progressed to deep apneic episodes requiring mechanical ventilation. During the following days, he showed serious neurological deterioration with intractable convulsions and coma, after 72 hours of admission. The cerebrospinal fluid (CSF) examination was normal. There were significantly elevated levels of serum ammonia $(761 \mu \mathrm{mol} / \mathrm{L})$ and plasma and urine citrulline (plasma: $2212.4 \mu \mathrm{mol} / \mathrm{L}$ ), as well as elevated orotic acid levels in urine at 152 hours of life. Glycine levels in both CSF and plasma were normal; normal ratio of CSF/plasma glycine concentration (0.038) away our initial diagnosis of nonketotic hyperglycinemia, that is most frequently presented with hiccups. He had no specific urine odor. Cranial ultrasonography revealed diffuse cerebral edema. We used noninvasively amplitude-integrated electroencephalography (aEEG) for assessing cortical status; it showed 
normal trace at admission (Figure 1a) but on 6 day of life aEEG showed severe abnormal trace (Figure 1b). He was immediately treated with sodium benzoate and high caloric parenteral solution, low-protein diet, and peritoneal dialysis initially. We used peritoneal dialysis because hemodialysis is not available in our hospital. Despite intensive treatment, infant died on 9 day of life because of cardio-respiratory insufficiency.

\section{DISCUSSION}

Hiccup which is a part of development phase of fetus can be a nonspecific symptom infrequently associated with vagus and phrenic nerve irritation, brain stem disorders, metabolic disorders, and drugs/toxins. ${ }^{6-8}$

Hiccups occur with a sudden inspiration immediately followed by active closure of glottis $^{6}$. The involuntary contractions of the diaphragm and intercostal muscles of hiccups can be induced by affecting the afferent branch (vagus and phrenic nerve), central branch (brainstem, midbrain), and the efferent branch (phrenic nerve, glottis and intercostal muscles) of the hiccup reflex arc. $^{7}$

Multiple causes of hiccups have been proposed. Encephalopathy in association with hiccup is frequently seen as a helpful diagnostic sign in nonketotic hyperglycinemia. The association between the high levels of glycine and hiccup is described as follows. This enzymatic defect has been shown to occur in brain and elevation of brain glycine concentrations leads to accumulation of glycine in brain that is critical in the neurotoxicity. ${ }^{9}$ Hiccups, which likely represent a brain stem excitatory effect, may relate to the probable excitatory effect of glycine. Finally the excitatory effect may cause seizures, hyperexcitability, and hiccups. ${ }^{9}$ It typically manifests in the first hours to days of life with rapidly progressive neurologic symptoms similar to citrullinemia. ${ }^{9,10}$

We initially consider our patient was compatible with nonketotic hyperglycinemia at onset of illness because of high frequency of hiccups in association with nonketotic hyperglycinemia. The laboratory diagnosis of nonketotic hyperglycinemia is based on simultaneous determination of CSF and plasma glycine showing an abnormal CSF-to-plasma glycine ratio (above 0.08 ). ${ }^{10}$

Citrullinemia is a rare autosomal recessive inborn error of the urea metabolism, caused by argininosuccinic acid synthetase deficiency. ${ }^{1,2}$ The diagnosis of citrullinemia is based on biochemical analysis of blood, plasma, and urine revealing increased levels of ammonia, citrulline, glutamine, and orotic acid as described in our patient. ${ }^{1-4} \mathrm{~A}$ study of the EEG in affected infants may showed the burst-suppression pattern typical of neonatal hyperammonemia and a close correlation of the severity of the pattern on the EEG with both the degree of hyperammonemia and depression of level of consciousness. ${ }^{3}$ Similarly, aEEG showed diffusely suppressed background activity in our patient (Figure 1b).

Hypercitrullinemia itself is possibly also neurotoxic by the inhibition of aerobic glucose

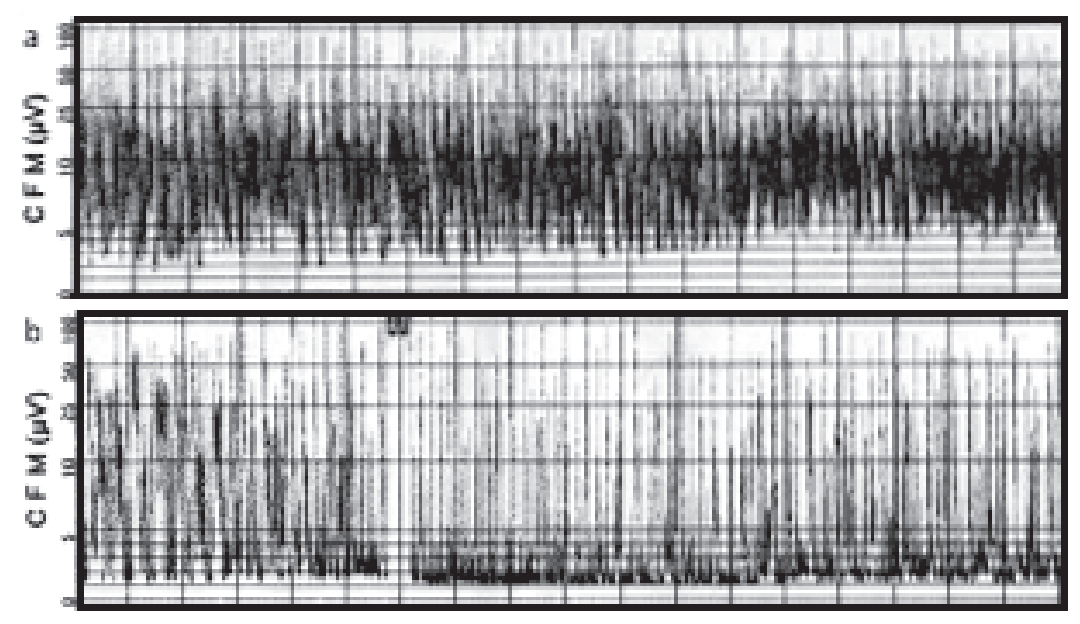


metabolism. ${ }^{4}$ If this disorder is not recognized early and treated promptly, death may follow cerebral edema, elevated intracranial pressure, and herniation as in our patient. ${ }^{1-4}$ There is no involvement of brainstem or role of excitatory effect of hypercitrullinemia. Neuropathology of hypercitrullinemia is associated with increased intracranial pressure that correlates with the severity of the neurological features ${ }^{1}$. In one case of neonatal citrullinemia, cortical-subcortical cystic infarcts were observed and thought to be related to decreased cerebral perfusion, secondary to elevated intracranial pressure. ${ }^{11}$

Brain stem infarction and tuberculoma was demonstrated in adult patients who were presented with intractable hiccup by magnetic resonance imaging. ${ }^{12}$

We think the cause of hiccup in citrullinemia may be due to brain stem infarction in association with increased intracranial pressure. But we could not show it by imaging studies because of clinical status rapid deterioration of our patient.

Among the weaknesses, our case was diagnosed by biochemical tests and not by enzyme and molecular biology tests. This may have some significance as there are forms of citrullinemia (associated with specific genotypes) that debut in the first years of life with liver failure.

Newborn screening is a public health program designed to screen infants shortly after birth for a list of conditions that are treatable, but not clinically evident in the newborn period. These potentially detectable diseases could be diagnosed early in life by the expanded newborn screening at the place of birth. ${ }^{13}$ Newborn screening is useful only in cases where clinical manifestations appear later. For early and severe clinical forms as with our patient, newborn screening is not useful.
To the best of our knowledge, encephalopathy in association with persistent hiccups in the neonatal period has not been reported with citrullinemia. It is possible that hypercitrullinemia may lead persistent hiccups in association with encephalopathy. Citrullinemia may be thought in the differential diagnosis of inborn errors of metabolism accompanied by hiccups.

\section{REFERENCES}

1. Wayenberg JL, Vermeylen D, Gerlo E, Pardou A. Increased intracranial pressure in a neonate with citrullinaemia. Eur J Pediatr 1992;151(2):132-3.

2. Sanjurjo P, Rodríguez-Soriano J. Management of neonatal citrullinemia. J Pediatr 1993;123(5):838-9.

3. Clancy RR, Chung HJ. EEG changes during recovery from acute severe neonatal citrullinemia. Electroencephalogr Clin Neurophysiol 1991;78(3):222-7.

4. Bireley WR, Van Hove JL, Gallagher RC, Fenton LZ. Urea cycle disorders: brain MRI and neurological outcome. $\mathrm{Pe}$ diatr Radiol 2012;42(4):455-62.

5. Smith HS, Busracamwongs A. Management of hiccups in the palliative care population. Am J Hosp Palliat Care 2003;20(2):149-54.

6. Madu AE, Oliver L. Non-ketotic hyperglycinaemia: case report and review of medical literature. J Matern Fetal Neonatal Med 2013;26(5):537-9.

7. Friedman NL. Hiccups: a treatment review. Pharmacotherapy 1996;16(6):986-95.

8. Loft LM, Ward RF. Hiccups: a case presentation and etiologic review. Arch Otolaryngol Head Neck Surg 1992;118(10):1115-9.

9. Tada K, Kure S. Non-ketotic hyperglycinemia: Molecular lesion, diagnosis and pathophysiology. J Inherit Metab Dis 1993;16(4):691-703.

10. Hoover-Fong JE, Shah S, Van Hove JL, Applegarth D, et al. Natural history of nonketotic hyperglycinemia in 65 patients. Neurology 2004;63(10):1847-53.

11. Martin JJ, Farriaux JP, De Jonghe P. Neuropathology of citrullinemia. Acta Neuropathol 1982;56(4):303-6.

12. al Deeb SM, Sharif H, al Moutaery K, Biary N. Intractable hiccup induced by brainstem lesion. J Neurol Sci 1991;103(2):144-50.

13. Mak CM, Lee HC, Chan AY, Lam CW. Inborn errors of metabolism and expanded newborn screening: review and update. Crit Rev Clin Lab Sci 2013;50(6):142-62. 